\title{
Informierte Welt - bessere Welt? Probleme des Überangebots von Information*
}

\author{
von Michael Schmolke
}

Im Jahre 1966 erschien in der Bundesrepublik Deutschland ein Buch, das schnell ein Bestseller wurde. Das lag nicht nur daran, daß der Autor ein geachteter Wissenschaftler war, der trotzdem verständlich zu schreiben wußte, sondern auch daran, daß das Thema genau zu den fortschrittsgläubigen Überlegungen einer Gesellschaft paßte, die einerseits von ihrem Erfolg überzeugt war und andererseits über ihre Zukunft nachzudenken begann. Es handelt sich um Karl Steinbuch: Die informierte Gesellschaft. ${ }^{1}$ In diesem Buch wurden "Geschichte und Zukunft der Nachrichtentechnik" behandelt. Steinbuch, der von Hause aus nicht Philosoph, sondern Professor für Nachrichtenverarbeitung und -übertragung an einer Technischen Universität ist, schildert zutreffend die informationstechnischen und -theoretischen Gegebenheiten der Vergangenheit und Gegenwart. Wo er sich der Zukunft zuwendet und "Gedanken zur langfristigen Prognose" vorträgt, äußert er zwar auch einige Skepsis, - im Grunde aber ist er vom Beginn einer neuen, durch die "Wissenschaftsexplosion" eingeleiteten Menschheitsepoche überzeugt. Er wertete diese Entwicklung positiv. Zum Beispiel hält er einen Prozeß der „Ent-Ideologisierung“ für notwendig und möglich, und die Haupt-Bedingung, die er für ein neues Zeitalter stellt, ist neben der EntIdeologisierung eine "neue Ethik", die ideologiefrei aufgebaut werden müsse.

Aber der Fortschritt der Menschheit scheint ihm in greifbare (und machbare) Nähe gerückt:

„Auch auf die Gefahr hin, pathetisch zu wirken, möchte ich doch die Vermutung aussprechen, daß der Mensch an einer entscheidenden Wende steht: Nämlich am Übergang vom irrationalen, vorwissenschaftlichen Zeitalter zum rationellen wissenschaftlichen Zeitalter. Vier Vorgänge wirken gemeinsam in dieser Richtung:

Die Entdeckung der Psychoanalyse, daß das Unterbewußte im Menschen rational analysierbar ist,

die Entdeckung der Kybernetik, daß zur Erklärung geistiger Vorgänge keine Voraussetzungen gemacht werden müssen, welche über die Physik hinausgehen, 
die Konfrontation verschiedener Kulturen durch die modernen Verkehrs- und Kommunikationsmittel, und

die politische Notwendigkeit, angesichts der Nuklearwaffen Ideologien abzubauen. ${ }^{\star 2}$

Steinbuch ist inzwischen skeptischer geworden ${ }^{3}$, aber sein Buch wirkte damals auf viele Menschen, vornehmlich auf Politiker, auch auf junge Wissenschaftler, wie das Programm einer neuen, kybernetisch inspirierten Aufklärung. Auch viele Wissenschaftler im Bereich der Kommunikationsforschung und der Massenkommunikationslehre hatten eine ähnliche Grundhaltung. Der niederländische Publizistikwissenschaftler Hendricus Johannes Prakke z. B. setzte zwar ebenfalls ethische Maßstäbe für kommunikatives Handeln, glaubte aber auf der anderen Seite eine Art von Selbst-Regulierung im massenkommunikativen Prozeß nachweisen zu können: Wenn man nur den öffentlich vermittelten Informationen in reichlicher Menge freien Lauf lasse, so werde die Gesellschaft in jedem Falle ihren Nutzen daraus ziehen; den Sinn von Publizistik sah Prakke darin, daß sie in der Lage sei, „Kulturwandel “ nicht nur zu begleiten, sondern auch zu bewirken - manchmal sogar auch „die großen Kulturwenden " mitzubewirken."

Während im 19. Jahrhundert und noch im ersten Drittel des 20. Jahrhundert, wenn von den damaligen Massenmedien die Rede war, die Sorge um die wabrheitsgemäße Information im Vordergrund stand, hielt man nach dem Zweiten Weltkrieg eine möglichst umfassende Information für wichtig. Ich fasse die Stimmung jener demokratisch-fortschrittsgläubigen Jahre in dem etwas übertrieben zugespitzten Satz zusammen: Je mehr Informationen, je breiter das Angebot, desto schneller marschiert der Fortschritt. Die rapide Entwicklung der Nachrichtentechnik in jener Zeit (Transistor, transistorisierte Computer, Koaxialkabel, Nachrichtensatelliten) ließ technische Verwirklichungen in greifbare Nähe rücken und damit auch die sozialen Hoffnungen glaubwürdig erscheinen. Erst Ende der 1960er Jahre fragte man wieder nach der "Richtigkeit" von Informationen, dieses $\mathrm{Mal}$ jedoch nicht im ethischen, sondern im politischen Sinne. Die These von der „Manipulation durch Massenmedien“ beherrschte die populärwissenschaftliche und politische Diskussion. Jetzt sprach man nicht mehr von der Menge des Informationsangebotes, sondern von der politischen Notwendigkeit, daß es vielfältig sein müsse. Beides bezieht sich auf unser Thema: Sowohl Vorstellungen der technologischen und kommunikationsphilosophischen Fortschrittsgläubigkeit (ungehindertes, möglichst breites Angebot) als auch Forderungen der Kommunikationspolitik (Vielfalt) endeten bei dem Satz, daß eine informierte Gesellschaft eine bessere sein werde, - global gesprochen: Informierte Welt - bessere Welt. Ich habe ein Fragezeichen zu setzen und zu begründen.

\section{Einige Daten und Trends}

Es ist eine Tatsache, daß in beinahe allen Ländern der Erde, die über freie, d. h. nicht der staatlichen Planung und Kontrolle unterworfene Massenkommunikationseinrichtungen verfügen, rein quantitativ von Jahr zu Jahr mehr „Informationen “ angeboten werden. ${ }^{5}$

Die Buchproduktion wächst, die Zeitungen und Zeitschriften werden, von den kurzen wirtschaftlichen Rezessionsphasen abgesehen, immer umfangreicher, die Fernsehprogramme erfassen immer mehr Stunden des Tages, viele Rundfunkanstalten strahlen drei oder vier verschiedene Programme aus, sowohl beim Hörfunk als auch beim 
Fernsehen. Ich begnüge mich damit, ein Beispiel mit Zahlen zu belegen: Die neun regionalen Rundfunkanstalten der Bundesrepublik Deutschland sind in der „Arbeitsgemeinschaft der öffentlich-rechtlichen Rundfunkanstalten der Bundesrepublik Deutschland" (ARD) zusammengeschlossen. Diese sogenannten ARD-Anstalten produzieren je drei Hörfunkprogramme ${ }^{6}$, ferner gemeinsam das Programm des "Deutschen Fernsehens" ( $=$ „erstes Programm“) sowie in fünf Arbeitsgemein-

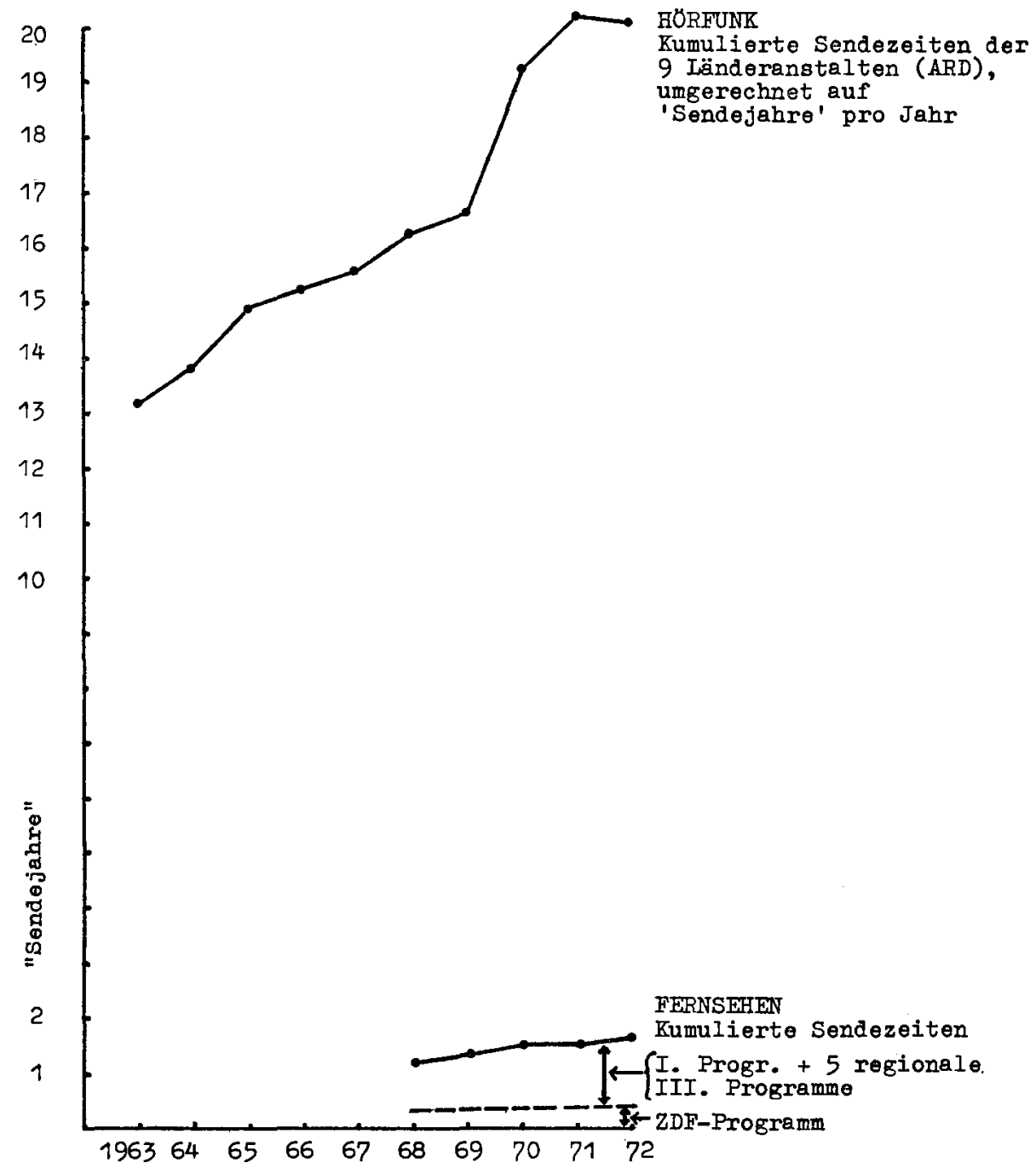

schaften fünf regionale Fernsehprogramme. Zählt man alle von den ARD-Anstalten gesendeten Programm-Minuten zusammen, so stellt man fest, daß in summa in dem einen Jahr 1972 20,02 Jahre Hörfunkprogramm ausgestrahlt wurden und dazu 1,28 Jahre Fernsehprogramm. ${ }^{7}$ Das heißt: Hätte man in der Bundesrepublik Deutschland nur einen Sender, der nur ein Programm ausstrahlen dürfte, so wäre er mit den Hörfunksendungen des Jahres 1972 (allein der neun Länder-Anstalten der ARD) 
für 20 Jahre eingedeckt, falls er täglich 24 Stunden sendete. Wollte er täglich 8 Stunden Fernsehprogramm ausstrahlen, so hätte er im kumulierten ARD-Fernsehprogramm des Jahres 1972 einen Vorrat für drei Jahre und zehneinhalb Monate.

Hörfunk und Fernsehen sind in der Bundesrepublik Deutschland schon lange etabliert, und dennoch ist ihr Angebot im letzten Jahrzehnt immer noch gewachsen: Wurden 197220 „Hörfunkprogramm-Jahre“ ausgestrahlt, so waren es zehn Jahre vorher (1963) "nur ${ }^{\star} 13,10$ und 1968 16,23. Erste und Dritte TV-Programme steigerten sich von 465.000 Sendeminuten $(=0,88$ Jahre) im Jahre 1968 auf 671.792 Minuten (= 1,28 Jahre) in 1972, das Programm des Zweiten Deutschen Fernsehens von 166.551 Sendeminuten $(=0,32$ Jahre $)$ im Jahre 1968 auf 197.448 Minuten $(=0,38$ Jahre) in 1972.

Wenn das Angebot wächst, so geht dies von den „Produzenten“ (Verlegern, Rundfunkanstalten, Redakteuren etc.) aus. Wie weit das Publikum die Ausweitung des Angebots nutzt, sich den Medien aussetzt, hängt von bestimmten Bedingungen ab. Dabei sei die Grundbedingung als gegeben vorausgesetzt: daß das Publikum bestimmte Bedürfnisse nach Information, Welt-Erklärung und Unterhaltung hat, die nur durch die Massenmedien befriedigt werden können. Ich spreche hier mehr von formalen Bedingungen: Das Publikum (die Rezipienten) muß, wenn es die Medien nutzen, ihre Angebote konsumieren will, haben:

a) Zeit

b) Geld

c) für die Nutzung der elektronischen Medien: eine bestimmte Geräteausstattung (Radio- und Fernsehgerät).

Die Art, wie diese drei Bedingungen als Faktoren zusammenwirken, ist in den einzelnen Ländern recht verschieden. In den Entwicklungsländern etwa bewirkt das Fehlen von "b“ und „c", daß die Massenmedien ihre gesellschaftlichen Funktionen oft nur in beschränktem Umfang erfüllen können. In den trend-bestimmenden Industrieländern aber haben fast überall in den letzten 20 Jahren die Menschen trotz Vollbeschäftigung mehr Freizeit und ein höheres Realeinkommen gewonnen, und sie haben ihre Haushalte in hohem Maße mit Radio- und Fernsehempfangsgeräten ausgestattet.

In der Bundesrepublik Deutschland waren Ende 1973 bei rund 60 Millionen Einwohnern 19,27 Millionen Radiogeräte und 17,27 Millionen Fernsehgeräte zugelassen; Ende 1967 waren es 18,56 Millionen (Hörfunk) und 13,67 Millionen (Fernsehen), Ende 1963: 17,06 Millionen (Hörfunk) und 8,37 Millionen (Fernsehen). (In anderen Industrienationen haben sich die Zahlen ähnlich entwidkelt.) ${ }^{8}$

Wollen und können die Menschen aber auch einen immer größeren Anteil ihrer freien Zeit für die Rezeption von Medien-Angeboten zur Verfügung stellen? Wie teilen sie ihr "Zeitbudget ${ }^{*}$ ein? 1964 haben die deutschen Umfrage-Institute DIVO und Infratest in einer gemeinsamen Untersuchung nicht nur nach "Nutzungsdauer", sondern auch nach „Medianutzung und Freizeit" gefragt. Damals verbrachte der deutsche (statistisch ermittelte) Durchschnittsbürger täglich 3:08 Stunden, indem er sich mit Fernsehen, Radiohören und Zeitunglesen beschäftigte; knapp zwei Drittel davon fielen in seine Freizeit im engeren Sinne. 1970 war die gesamte den Medien gewidmete Zeit auf 3:34 Stunden gestiegen, d. h. um 13,8\% ${ }^{10}$ (Es verwundert nicht, daß das Fernsehen dabei das stärkste Wachstum hatte: um 61,4\% von 70 auf 113 
Minuten täglich.) Der für die Massenmedien aufgewandte Anteil des Zeitbudgets wird jedoch noch weiter wachsen. Aus den Niederlanden, einem in vieler Hinsicht progressiven Land, wurde schon für 1971 von einer täglichen Mediennutzungsdauer von fünf Stunden berichtet. ${ }^{11}$ Andererseits ist hier eine natürliche Wachstumsgrenze gegeben: Der Tag hat nur 24 Stunden, und acht davon braucht man für den Schlaf. Es bleibt aber die Frage offen: Wie teilt der Rezipient seine drei oder höchstens fünf "Medienstunden" sinnvoll ein, wenn ihm Angebote für die fünf- oder zehnfache Zeit vorliegen?

Man könnte einwenden: Auf dem Gebiet der Presse sorge die anhaltende Pressekonzentration für eine geringere Angebotslast. Aber das ist ein Trugschluß. Zum einen bleibt die durchschnittliche tägliche Lesezeit für Tageszeitungen seit Jahren fast gleich (in der Bundesrepublik Deutschland: ca. 30 Minuten), zum anderen sinkt zwar die Zahl der redaktionell voll selbständigen Tageszeitungen, aber die Tageszeitungsgesamtauflagen steigen, - in Deutschland trotz Marktsättigung von 17,65 Millionen (1971) auf 18,5 Millionen (1973), in Osterreich von 2,18 Millionen (1971) auf fast 2,3 Millionen Exemplare (1973) ${ }^{12}$ täglich. (Vgl. dazu die graphische Darstellung Nr. 2; nur für die Bundesrepublik Deutschland.)

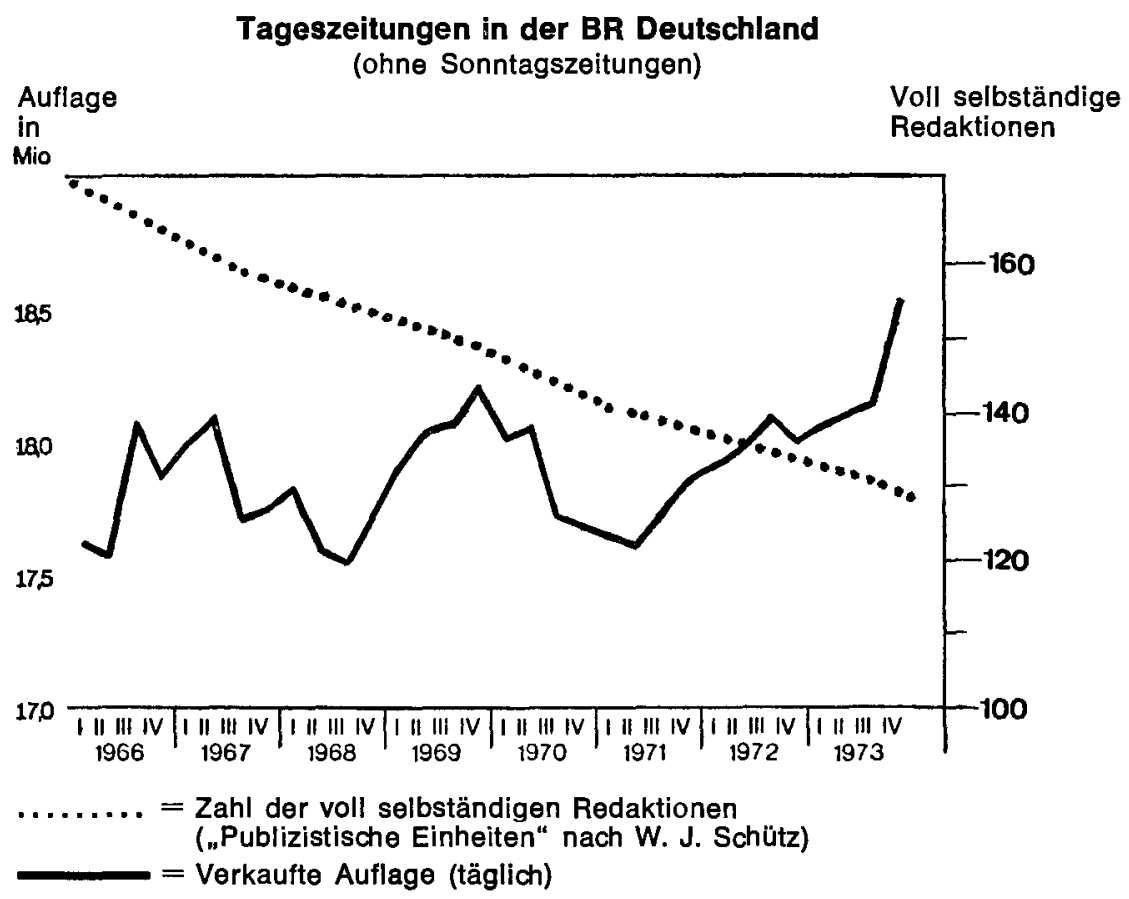

Während der Pressemarkt Anzeichen von Sättigung zeigt, scheint auf dem Gebiet der audiovisuellen und elektronischen Medien eine Expansion bevorzustehen. Dies gilt weniger für die Ton-Bild-Kassette und -Platte als für das sogenannte KabelFernsehen. Der Aufbau des Kabel-Fernsehens hat in den USA etwa um 1950 begonnen; 1952 gab es 70 Kabelsysteme mit 14.000 Teilnehmern, 19722.770 Systeme 
mit 6 Millionen Teilnehmern; 1973 wurden 5.663 Gemeinden von 2.966 KabelSystemen bedient. Von diesen Systemen können nicht nur Fernsehprogramme in größerer Auswahl und besserer Qualität ins Haus gebracht werden, sondern elektronisch vermittelte Informationsangebote verschiedenen Inhalts. Die heutigen Anlagen bieten etwa 12 Kanäle, die neuesten 20 , die künftigen 20 bis $40 .{ }^{13}$ In absehbarer Zeit wird es technisch möglich sein, nicht nur Angebote zu wählen und $\mathrm{zu}$ empfangen, sondern auch selbst (Rezipient als Kommunikator) in der Gegenrichtung fragend oder sagend tätig zu werden. Das bedeutet: Die bisher schon hochgradig belastete Medienaufnahme-Kapazität wird in Zukunft durch die Chance (aber auch die Notwendigkeit), zwischen viel mehr Möglichkeiten auszuwählen, noch stärker belastet werden. Es ist eine offene Frage, ob sich der normale, nicht speziell vorgebildete Rezipient der neuen Informationsbeschaffungsmöglichkeiten mit der gleichen Selbstverständlichkeit wird bedienen können wie heute des Telefons.

Fazit: Die Massenmedien präsentieren heute schon Informationsangebote in einem so großen Umfang, daß kein einzelner Konsument sie voll ausschöpfen kann. ${ }^{14}$ Künftige, im Aufbau befindliche Kommunikationssysteme werden noch mehr anbieten, während auf der Seite des Rezipienten weder die Aufnahmekapazität noch die Auswahlfähigkeit beliebig gesteigert werden kann.

\section{Medienkommunikation als Existenzbedingung moderner Gesellschaften}

"Information ist Anfang und Grundlage der Gesellschaft." Diese alltäglich klingende Feststellung Karl Steinbuchs (a.a.O., 7) weist auf ein Dilemma hin: Obwohl wir noch nicht richtig wissen, wie wir mit der Uberfülle von Informationen umgehen sollen, können wir ohne sie nicht leben.

Es ist evident, daß moderne Industriegesellschaften, wenn sie eine gewisse, historisch feststellbare Schwelle der Bevölkerungsdichte und der Warenproduktion erreicht haben, auf technische Kommunikationsmittel und -hilfsmittel (im weiteren Sinne, einschließlich der technischen Verkehrs- und Transportmittel sowie der Informationsspeicherungs-, -transport- und -verarbeitungsmittel) nicht mehr verzichten können. Das heißt: Diese Mittel sind für so beschaffene Gesellschaften existenznotwendig. Ich gehe dabei von der Annahme aus, daß die gesellschaftliche Entwicklung mit

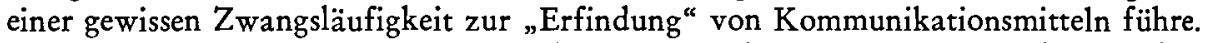
Vor fast 130 Jahren konstatierte Robert Prutz: die Ausweitung der literarischen Produktion habe nicht stattgefunden, weil im 15. Jahrhundert die Buchdruckkunst erfunden worden sei, sondern diese Kunst sei erfunden worden, ,weil der geistige Zustand der Welt ein solches Mittel allgemeinster Mittheilung nöthig machte ${ }^{\text {". }}{ }^{15}$ Das gleiche gilt u. E. für alle technischen Kommunikationsmittel einschließlich der Verkehrsmittel. Deren Entwicklung und Verbesserung hat die Zahl der Kommunikationschancen ins kaum noch Meßbare und auch die Zahl der wirklich vollzogenen Kommunikationsvorgänge ins allenfalls noch repräsentativ Darstellbare vermehrt.

Man ist versucht, für die Bedingungen in Entwicklungsländern Einschränkungen zu machen. Millionen von Menschen leben dort nach wie vor ohne die Kommunikationszwänge, denen wir im Hinblick auf die Aufnahme von Informationen ständig ausgesetzt sind. Aber da diese Länder wegen des großen Vorsprungs Europas und Amerikas praktisch gezwungen sind, in Wirtschaft und Technik den Mustern der Verwestlichung weitgehend zu folgen, werden sie den Bereich der Kommunikationstechnologie und damit auch der Kommunikationskultur nicht aussparen können. Sie 
bedienen sich anderer Symbole, anderer Zeichensysteme und anderer Formen, aber eines Tages werden auch sie - mag man es noch so sehr beklagen - der „Informationslawine ${ }^{\alpha 16}$ gegenüberstehen.

\section{Einige kritische Beobachtungen}

Die heute in Nachrichtenagenturen oder Rundfunkanstalten täglich umgesetzten Mengen von Wörtern sind bereits sehr groß: Die Deutsche Presseagentur (dpa) empfängt täglich 450.000 bis 500.000 Wörter und gibt davon 40.000 bis 45.000 weiter. Auch der Westdeutsche Rundfunk Köln, eine der neun regionalen deutschen Rundfunkanstalten, hat einen täglichen Eingang von fast 400.000 Wörtern. Die (kleine) österreichische Austria Presse Agentur (APA) hat 1973 13,6 Millionen Wörter über Fernschreiber an Zeitungen und Rundfunk weitergegeben. Wirklich bemerkenswert sind aber nicht so sehr die absoluten Zahlen als das, was das Schlagwort "Informationslawine" meint: die anscheinend unaufhaltsame Vermehrung des Informationsumsatzes: „Eine Faustregel sagt, daß sich das Informationsvolumen ungefähr alle zehn Jahre verdoppelt. Das heißt, von 1960 bis 1980 haben wir insgesamt eine Vervierfachung dessen zu erwarten, was wir $1960 \mathrm{zu}$ bewältigen hatten. ${ }^{{ }^{17}} \mathrm{M}$. Scheele hat aus verschiedenen Quellen konkrete Anhaltspunkte aus dem Bereich der wissenschaftlichen Kommunikation zusammengetragen:

Für das Jahr 1800 wird die Zahl der wissenschaftlichen Fachzeitschriften auf 100 geschätzt, für 1900 auf 10.000, für 1965 auf mehr als 100.000. Geht man von der Annahme aus, man könne pro Stunde 30 Seiten lesen, und das acht Stunden pro Tag, "so konnte ein Physiologe im Jahre 1879 seine Zeitschriftenliteratur bei einem Tagespensum von 240 Seiten innerhalb von 37 Tagen und 4 Stunden ,bewältigen'. Für das Jahr 1930 betrug die Lesezeit bei ausschließlichem Literaturstudium pro Jahr schon mehr als ein Jahr, im Jahre 1940 betrug sie mehr als zwei Jahre, zur Zeit dürfte sie noch wesentlich höher liegen. ${ }^{{ }^{18}}$

Je größer die Informationsmengen werden, desto stärker wird auch die Kraft ihrer Strömung. Das heißt: Die Anforderungen an jene, die professionell auswählen müssen, die "gatekeeper", werden immer höher. Dank der gatekeeper-Forschung ${ }^{10}$ wissen wir viel über die Ausübung dieser Berufsrolle, wir wissen aber auch, daß es keine sicheren Maßstäbe für eine "gerechte" Nachrichten-Selektion gibt und daß das tatsächliche Handeln der gatekeeper zum Teil von anderen Argumenten als vom Inhalt der Nachrichten bestimmt wird. ${ }^{20}$ Auch gibt es - jedenfalls für Journalisten - keine spezialisierte Ausbildung, die eine korrekte Selektion erleichtern würde. Die gatekeeper wissen jedoch, daß ihnen auf der Seite des Publikums wiederum selektives, von bestimmten Bedürfnissen gesteuertes Verhalten gegenübersteht, und in mancher Hinsicht orientieren sie sich daran.

Daß sich Rezipienten selektiv verhalten, ist in einer Reihe von Untersuchungen festgestellt und erhärtet worden. Sie tun es besonders dann, wenn es um ihr inneres Gleichgewicht, ihre seelische Harmonie, um die Vermeidung von Dissonanzen geht. ${ }^{21}$ Dieser Hinweis soll sich hier nur auf die Frage beziehen, ob der mit Selektion oder „selektiver Wahrnehmung" beschriebene Selbstschutz-Mechanismus ausreicht, um auch mit dem immer stärkeren Anprall immer größerer Informationsmengen (als Angebote) fertig $\mathrm{zu}$ werden oder ob nicht auf die Dauer geradezu eine Abschirmung des Individuums die Folge sein wird. Schon heute scheint es doch häufig so zu sein, 
daß der Rezipient eine Tageszeitung liest, sich mit einer Unterhaltungszeitschrift begnügt, zwischen nur zwei Fernsehprogrammen schwankt und den Hörfunk nicht selten nur zufällig einschaltet? Aus Abschirmung könnte Abstumpfung werden.

Schließlich stellt sich die Frage nach Kontrolleinrichtungen für die öffentliche Kommunikation. Wo immer durchfließende Mengen zu groß werden, richtet man Kontrollen und Regelungsinstrumentarien ein: Dämme, Wehre und Schleusen für das Wasser, Sicherungen für den elektrischen Strom, Blenden für das Licht. Statt "für" könnte man auch "gegen" sagen. Darin wird das Problem der Kontrolle von Informationsströmen deutlich. Die Beseitigung ihrer Behinderung durch die Herstellung der Pressefreiheit hat die Entstehung der modernen Demokratie ermöglicht. Wer wollte es heute riskieren, die Kontrolle (im Sinne von Regulierung, auch Einengung) des Informationsstroms übergeordneten Instanzen anzuvertrauen? Es ist bekannt und nachweisbar, daß den einzelnen Rezipienten am Ende ohnehin nur Fragmente aus dem Gesamt-Angebot erreichen, aber es ist etwas anderes, ob die Auswahl dieser Fragmente dem Zufall und der Selektion des Einzelnen überlassen bleibt, oder ob ein "Großer Bruder" (George Orwell) die Vor-Auswahl trifft.

So paradox es klingen mag: In dem Stadium, das die liberalen, parlamentarischdemokratischen Gesellschaften des Westens zur Zeit erreicht haben, könnte gerade die Forderung nach noch mehr "Demokratisierung " den Großen Bruder heraufbeschwören. Denn die Beteiligung von immer mehr Bürgern an immer mehr Entscheidungsvorgängen ist in der Regel nur über die Einrichtung immer neuer „Kollektive“ möglich. Dies gilt auch für den Bereich der öffentlichen sozialen Kommunikation. Es ist ein Trugschluß, zu glauben, daß die Einbeziehung von immer mehr Mit-Redenden in Pseudo-Gatekeeper-Akte die „kommunikative Gleichheit“ befördern könnte, ohne $\mathrm{zu}$ Lasten der publizistischen Freiheit (aktiv und passiv) zu gehen. Helmut Schelskys

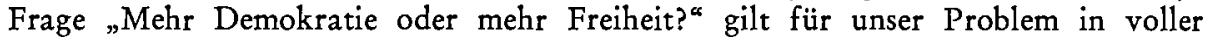
Tragweite. Der von ihm kritisierte Abbau der (freiheitsichernden) Gewaltenteilung ${ }^{22}$ brächte nicht nur keine Lösung der Aufgabe, immer größere Informationsmengen in immer schnellerem Fluß einigermaßen korrekt an immer mehr Abnehmer zu vermitteln, sondern er würde das Dilemma verschlimmern, indem er nach außen hin Scheinlösungen präsentieren könnte, die in Wirklichkeit nur verschleiern. Wenn es so ist, daß durch Gewaltenteilung „ein viel höherer Schutz des einzelnen gegenüber allen Machtkonstellationen gegeben" ist, „als je eine auch mehrheitlich bestimmte Demokratie gewähren kann ${ }^{\alpha 23}$, dann wäre für Gewaltenteilung, und zwar zunächst pauschal ${ }^{24}$ zugunsten der Könner unter den Machern zu plädieren. Die Verantwortung der Gesamtgesellschaft hätte sich demnach in erster Linie auf die Gewährleistung und Kontrolle der Könnerschaft zu erstrecken: gegenüber Kommunikatoren (Stichwort: Journalistenausbildung) und Rezipienten (Stichwort: kommunikative Kompetenz). ${ }^{25}$ Das Handeln aber, sowohl das publizierende als auch das rezipierende, sollte in freien Gesellschaften der freien Entscheidung des Einzelnen unterworfen bleiben, selbst wenn diese Entscheidungschance, wie viele Kritiker behaupten, vielfach nur in minderer Qualität wahrgenommen wird.

Am Ende steht also, wie bei so vielen Problemen, die durch den Sachbereich der öffentlichen gesellschaftlichen Kommunikation aufgeworfen werden, die Frage nach einer individuellen, aber auch sozialen Ethik des kommunikativen Verhaltens. ${ }^{20}$ Dabei wird man die Beobachtung einbeziehen müssen, daß nicht nur die Angebotsquantitäten gestiegen sind und weiter steigen, sondern daß auch die Entfaltung der Medien - 
kein Medium "stirbt" ! - Veränderungen im Kommunikationspotential und -verhalten unserer Gesellschaft bewirkt hat: Das fing an beim gedruckten Buch, das das Vorlesen fast überflüssig und den Einzel-Leser massenhaft möglich machte ${ }^{27}$, und es hört - vorläufig - auf beim Fernsehen, von dem wir mindestens jene Wirkung zuverlässig kennen: daß es in der Lage ist, bisher ungekannt große Sektoren aus dem Zeitbudget der Zeitgenossen für sich herauszuschneiden ${ }^{28}$ und bei besonderen Ereignissen mehrere hundert Millionen Menschen vor den Bildschirm zu ziehen. Diese Zeit fehlt irgendwo im Kommunikationszeitbudget der Menschen, - sie ist, wie man in Osterreich sagt, umgewidmet. Und auch die umgewidmete Intensität der Zuwendung dürfte kaum beliebig reproduzierbar sein. Sollte aufgrund dieser Entwicklung die expandierende Medienzuwendung zum "Sparen" in der interpersonalen Kommunikation führen - und einige Anzeichen deuten darauf hin - so gewinnt unsere Frage doppelte Bedeutung: informierte Welt - bessere Welt?

Anmerkungen:

* Diesem Beitrag liegt eine Vorlesung zugrunde, die der Mitherausgeber von „Communicatio Socialis" am 15. Mai 1974 im Rahmen des "Programa para Graduados Latinoamericanos en Medios Informativos" vor lateinamerikanischen Journalisten bzw. Absolventen einschlägiger Studien an der Universität von Navarra in Pamplona gehalten hat. Das Fortbildungsprogramm wird von der Bischöflichen Aktion Adveniat gefördert. (Vgl. CS $6: 1973$, S. $157-159$.)

1. Erste Ausgabe Stuttgart 1966; ab 1968 in mehreren Auflagen als rororo-Taschenbuch, hier benutzt in der Ausgabe Reinbek 1972.

2. A.a.O., S. 240 f.

3. Vgl. z. B. zuletzt seinen Vortrag „Kommunikation und Politik“ auf dem CDU/CSUKongreß zur Fortentwicklung und Sicherung der Presse- und Informationsfreiheit 30./31. Mai 1974 in München.

4. H. Prakke: Vom Sinn der Publizistik, Assen/Niederlande 1965, S. 21 - Die Mitwirkung der Massenkommunikation beim sozialen Wandel ist inzwischen in einer Reihe von empirischen Studien beobachtet worden und erklärt worden. (Man vergleiche z. B. die Arbeiten des Amerikaners D. Lerner, einen Uberblick gibt W. Schramm: Mass Media and National Development, Stanford 1964;) die jüngste Spezialstudie (in deutscher Sprache) ist die Dissertation von Rosario Gomez SJ: Massenkommunikation und Bewußtseinsbildung der Inder, Diss. Münster 1973.

5. Einige grobe quantitative Anhaltspunkte geben z. B. die globalen Anspruch erhebenden Statistischen Jahrbücher der UNESCO. - Den Begriff "Information " verwende ich hier in einem weiten, andererseits durch das Kriterium der allgemeinen Zugänglichkeit aufgrund von "Veröffentlichung “ eingegrenzten Sinne: zur Bezeichnung aller von den publizistischen Medien (im weitesten Sinne) angebotenen Inhalte (einschließlich der Kommentierung und Unterhaltung); dabei gehe ich von der Hypothese aus, daß es letztenendes der Rezipient ist, der entscheidet, aus welchen zu rezipierenden Inhalten er, ungeachtet der Intention des Kommunikators, durch Definition und Interpretation informatorischen Nutzen zu ziehen gewillt ist. Zur Diskussion des Informationsbegriffs: G. Wersig: Information - Dokumentation - Kommunikation, München-Pullach 1971, S. 25-74.

6. Beim Ersten Programm teilen sich WDR und NDR in die ihnen zur Verfügung stehenden Sendemöglichkeiten, bei den Dritten Programmen wirken z. B. NDR und SFB zusammen. Genaue Aufschlüsselungen vermitteln die „ARD-Jahrbücher", die im Verlag des Hans-Bredow-Instituts Hamburg erscheinen.

7. In Bezug auf die Programminhalte stimmt diese Addition nicht ganz: es sind nicht alles verschiedene Inhalte, weil die Anstalten ein gewisses Maß von Sendungen voneinander übernehmen und weil einzelne Sendungen gelegentlich wiederholt werden. 
8. Genaue Daten dazu liefert regelmäßig die Zeitschrift „Rundfunk und Fernsehen“ (Hamburg) in ihrer Rubrik, Chronikc.

9. DIVO und Infratest: Massenkommunikation. Ergänzung oder Konkurrenz der Massenmedien, Teil 1, in: ARD (Hrsg.): Rundfunkanstalten und Tageszeitungen, Dokumentation 4, o.O., 1966, S. 1-63, hier S. 12.

10. Die Nutzung der Freizeit, in: „ZV+ZV“, Bonn - Bad Godesberg, Nr. 20/1972, S. 914.

11. [J. G. Stappers und] W. J. M. Evers: Seelsorge im Abseits der Massenkommunikation, in: "Communicatio Socialis“ (CS) 6:1973, S. 103.

12. Vgl. „ZV+ZV“, Nr. 14/1974, S. 372 ff. und „Salzburger Nachrichten“ vom 4. April 1974. Zur Entwicklung der Pressekonzentration in der BR Deutschland vgl. den Bericht der Bundesregierung über die Lage von Presse und Rundfunk in der Bundesrepublik Deutschland (1974) vom 15.5.1974 (Bundesdrudksade 7/2104), insbesondere S. $5 \mathrm{ff}$. und $11 \mathrm{ff}$.

13. Vgl. die informativen Beiträge in der Zeitschrift "Media Perspektiven“, Frankfurt/Main, zuletzt: USA: Neues Konzept zum Kabelfernsehen, in Nr. 2/1974, S. 60-65, und E. W. Fuhr: Die Entwidklung des Kabelfernsehens und seine möglichen Auswirkungen auf Presse und Rundfunk, in Nr. 5/1974, S. 195-207; sowie H. J. Kleinsteuber: Zur Entwicklung des Kabelfernsehens am Beispiel der USA. Vortrag beim Kongreß der Deutschen Vereinigung für politische Wissenschaft, Hamburg, Oktober 1973 (vervielf. Msk.).

14. „Wenn ich selbst als politisch interessierter Professor mit der Lektüre von vier Tageszeitungen, mit nicht mehr als einer Stunde Rundfunk- oder Fernsehteilnahme je Tag zufrieden sein muß - und mehr würde meine Teilnahme an der ,öffentlichen Diskussion" zu einem Teilberuf machen, der meinen Hauptberuf entschieden einschränken würde was erwartet man dann von der Aufnahmefähigkeit eines normalen, nicht intellektuellen Bürgers in Bezug auf Meinungsbildung durch Abwägung der ,öffentlichen politischen Diskussion'?" H. Schelsky: Publizistik und Gewaltenteilung, = S. 83-108 in: H. Schelsky: Systemüberwindung, Demokratisierung und Gewaltenteilung, München ${ }^{3} 1973$, hier S. 93.

15. R. E. Prutz: Über die Unterhaltungsliteratur insbesondere der Deutschen, in: "Literarhistorisches Taschenbuch", Hannover 3 (1845), S. 423-454, hier S. 438. - Ich beziehe mich hier auf meinen Aufsatz "Die Massenmedien im Dienst des Friedens?", in: R. Weiler/ V. Zsifkovits (Hrsg.): Unterwegs zum Frieden, Wien 1973, S. 581-599.

16. So der Titel eines populär-wissenschaftlichen Buches von U. Bischoff, Düsseldorf 1967.

17. U. Bischoff, a.a.O., S. 15 f.

18. M. Scheele: Wissenschaftliche Dokumentation, Schlitz/Hessen 1967, S. 11 f.

19. G. J. Robinson: 25 Jahre „Gatekeeper"-Forschung: Eine kritische Rückschau und Bewertung, in: J. Aufermann, H. Bohrmann, R. Sülzer: Gesellschaftliche Kommunikation und Information, Bd. 1, Frankfurt/Main 1973, S. 344-355.

20. Z. B. vom Zeitdrudk oder von der Betriebsform einer Zeitung. Vgl. Ch. Kristen: Nachrichtenangebot und Nachrichtenverwendung, Düsseldorf 1972.

21. Vgl. dazu F. Bledijan: Theoretische Ansätze über den Einfluß der präkommunikativen Einstellungsstruktur der Rezipienten auf Bildung und Änderung von Einstellungen, in: D. Prokop (Hrsg.): Massenkommunikationsforschung 2: Konsumtion, Frankfurt/Main 1973, S. 213-248.

22. H. Schelsky: Mehr Demokratie oder mehr Freiheit? S. 47-82 in: H. Schelsky: Systemüberwindung, Demokratisierung und Gewaltenteilung, München ${ }^{3} 1973$.

23. Ebenda, S. 57.

24. Nicht zu verwechseln mit jener ebenfalls von Schelsky an gleicher Stelle (S. 83-108: Publizistik und Gewaltenteilung) diskutierten Gewaltenteilung, die für die "freiheitliche Gestaltung der Informationsmacht ${ }^{*}$ erforderlich sei (S. 108).

25. Vgl. dazu D. Baacke: Kommunikation und Kompetenz, München 1973.

26. Für den individuellen Bereich versucht, pädagogisch orientiert, E. Bülow eine Antwort: Kommunikative Ethik, Düsseldorf 1972.

27. Genaueres dazu bei M. Schmolke: Die Massenmedien im Dienst des Friedens? (Vgl. Anm. 15), S. $590 \mathrm{f}$.

28. „Freizeitfresser Fernsehen“: vgl. E. Gehmacher, D. G. Graf, L. Spira: Buch und Leser in Osterreich, Hamburg 1974, S. 68 ff. 
According to many authors the quantitative and qualitative improvement of available public information automatically fosters the progress of humanity. Indeed the information supply of the industrialized countries increases steadily. The single recipient can consume only a part of the total information accessible to him. Here the limit is most probably already reached since some time ago, and the ability to select is most probably overcharged. On the other hand a selection must be possible for the recipient in order to respect his democratic freedom. The fact that there is too much information does not give the right to introduce further controls. Competence and responsibility of the professional communicator ("communications ethic") and also of the recipients have to be developed and strengthened.

\section{RÉSUME}

Beaucoup d'auteurs affirment que l'amélioration quantitative et qualitative de l'offre publique en informations encourage automatiquement le progrès de l'humanité. En effet, l'offre en informations continue de grandir, même dans les pays fortement industrialisés. Chaque receveur en particulier ne peut recevoir qu'une fraction de ce qui est a sa disposition dans son rayon d'écoute. La limite de la capacité d'assimilation doit être depuis longtemps franchie. Mais aussi, on exige trop de la capacité de choix. D'autre part, l'offre à partir de laquelle on peut choisir doit être maintenue à cause de la liberté démocratique. La connaissance du fait que dans l'ensemble on offre trop ne doit pas aboutir à la mise en place d'instances supérieures de contrôle. Au contraire, la compétence et la responsabilité („éthique communicative") des communicateurs professionnels, de même que celles des receveurs, doivent être encouragées et renforcées.

Zu Artikel: Westerbarkey

\section{R E S U M E N}

Muchos autores afirman que el perfeccionamiento cuantitativo y cualitativo de la oferta pública de informaciones contribuye automáticamente al desarrollo de la humanidad. De hecho aumenta constantemente la oferta informativa incluso en los países mas industrializados. Cada receptor personal hace uso solo parcial de cuanta información se pone a su disposición. Se rebasó con mucho el límite de capacidad receptora. También la capacidad de selección está saturada. Por otra parte, si se quiere libertad democrática, debe mantenerse una oferta, de la que cada cual pueda elegir. El reconocimiento de que la oferta resulta excesiva no debe conducir al establecimiento de controles. Por el contrario debe promoverse y robustecerse la competencia y responsabilidad (ética de la comunicación) de los profesionales de los medios de comunicación. 